\title{
Successful Renal Re-transplant in a Patient with Alport Syndrome: A Case Report
}

\author{
Mishra N Mahendra ${ }^{1 *}$, Indu Bansal ${ }^{2}$ and Vandana Lal ${ }^{3}$ \\ ${ }^{1}$ Baptist Christian Hospital, Tezpur, Assam, India \\ ${ }^{2} R G$ Stones Urology and Laparoscopy Hospital, Mumbai, India
}

${ }^{3}$ Dr Lal Path Labs Ltd., New Delhi, India

*Corresponding author: Mishra N Mahendra, Baptist Christian Hospital, Tezpur, Assam, India, Tel: 44-2890329241; E-mail: mnmishra@hotmail.com Received Date: August 20, 2018; Accepted Date: August 27, 2018; Published Date: September 03, 2018

Copyright: ( $) 2018$ Mahendra MN, et al. This is an open-access article distributed under the terms of the Creative Commons Attribution License, which permits unrestricted use, distribution, and reproduction in any medium, provided the original author and source are credited.

\begin{abstract}
This case report is of a sensitized patient with Alport syndrome, who received second kidney transplant from HLA-DQB1 incompatible living donor after excluding two unrelated donors. Four months after transplant he developed BK virus allograft nephropathy, from which he recovered and has excellent renal function-four years posttransplant. The findings highlight that HLA incompatible renal transplant can be performed successfully with prudent pre, and post-transplant evaluation complemented by suitable immunosuppression in sensitized recipients. It is presented on account of its rarity and is possibly the first documented case of re-transplant across a HLA-DQB1 barrier in India as defined by positive Luminex cross matches.
\end{abstract}

Keywords: Alport syndrome; Re-transplant; Luminex crossmatch; BK virus allograft nephropathy

\section{Introduction}

Alport syndrome is a rare heterogeneous group of X-linked (85\%) or autosomal recessive $(15 \%)$ disorder with a prevalence of 1:50000, which affects the glomerular basement membrane and most patients require renal replacement therapy $[1,2]$. Luminex crossmatch (LXM) has been used successfully for both pre and post -transplant evaluation of kidney recipients, including for monitoring response to immunosuppressants [3]. Here we report a case of HLA incompatible retransplant in which the recipient developed BK virus nephropathy but could be salvaged with prudent titration of immunosuppression and now four years post-transplant has normal serum creatinine.

\section{Case Report}

The 45-year-old male patient was one of six affected male children in extended joint family, first became symptomatic at the age of six years and received first renal transplant at the age of 24 , years which functioned for 20 years.

\begin{tabular}{|c|c|c|c|c|c|}
\hline Category & Test/ Method & Date & HLA-Class I & HLA-Class II & Remarks \\
\hline Donor 1 & R-SSO & $26 / 05 / 12$ & $A^{\star} 24,{ }^{*} 33 ; B^{*} 44,{ }^{*} 57$ & $\mathrm{DRB} 1^{*} 07$ & DQB1 not typed \\
\hline Cross match & $\mathrm{CDC}$ & June-2012 & $\mathrm{T}$ and $\mathrm{BCXM}$ negative & - & - \\
\hline- & Luminex (MFI) & $17 / 12 / 12$ & 918 & 923 & CDXM-negative \\
\hline- & Luminex (MFI) & $08 / 05 / 13$ & 1144 & 663 & Donor excluded \\
\hline SAB \& PRA ID I & Class I (MFI) & $\begin{array}{l}26 / 09 / 12 \\
09 / 05 / 13 \\
27 / 02 / 14\end{array}$ & $\begin{array}{l}\text { A24-DSA } 3711 \text { A24-DSA } 11613 \\
\text { A24-DSA } 8788\end{array}$ & $\begin{array}{l}\text { DRB1*07 negative } \\
\text { Not done }\end{array}$ & $\begin{array}{l}\text { Rise in DSA MFI PRA } \\
\text { ID(I) }-4237 \text { Jan } 14\end{array}$ \\
\hline Donor 2 & $\mathrm{CDC}$ & $20 / 05 / 13$ & $\mathrm{~A} 29,-; \mathrm{B} 7,-\mathrm{Bw} 6 ; \mathrm{Cw}-$ & DR10, 15; DR51 & No repeat SAB Class II \\
\hline HLA typing & $\mathrm{CDC}$ & $12 / 06 / 13$ & $\mathrm{~A} 29,-; \mathrm{B} 7,40,-\mathrm{Bw6}$;Cw- & Not done & Donor excluded \\
\hline Crossmatch & $\mathrm{CDC}$ & $11 / 06 / 13$ & TCXM negative & BRXM weak positive & (After donor exclusion) \\
\hline PRA ID I & Luminex (MFI) & $22 / 06 / 13$ & 353 & 6420 & DR15 3542, DR51 3127 \\
\hline
\end{tabular}

Table 1: HLA typing and antibody workup for two rejected donors.

None of the female siblings had any disease manifestation. He was on maintenance haemodialysis for four years before a suitable donor was identified. Comprehensive immunogenetic evaluation including
Human Leukocyte Antigen (HLA) typing on multiple samples and extensive antibody workup was carried out (Table 1). 
Page 2 of 3

\section{Methods}

The third donor was typed for eight loci (HLAABCDRB1DQA1DQB1DPA1DPB1) by reverse SSO (LIFECODES HLA SSO). HLA-ABDRB1 typing of the recipient was carried out by reverse-SSO. Additional typing for HLA-C and -DQB1 alleles was done on a second sample. Verification typing for eight loci was carried out HLA-ABCDRB1DQA1DQB1DPA1DPB1 on an additional sample in another laboratory, and all results were concordant. CDC crossmatch which was outsourced was performed, against all the three donors, by augmented Anti Human Globulin method. LXM was performed against all three donors as mentioned previously [3]. Phenotype bead assay was done for both HLA-Class I and II in Dr Lal Path Labs.

\section{Results}

HLA typing results of the donors and patient along with antibody profile are shown in Tables 1 and 2. Class I/II percentage panel reactive antibody (PRA) of $62 / 75 \%$ as detected by Luminex phenotype assay (LIFECODES Class I and Class II ID). CDC crossmatch was negative, against the first donor, but LXM tested positive for Class I and II donor specific antibodies- the former was substantiated by single antigen bead assay leading to exclusion of the first donor. DSA was detected against the second donor by CDC crossmatch which was weakly BCell positive and strongly positive for Class II antibodies by LXM (LIFECODES DSA) leading to exclusion of the second donor (Table 2).

SAB assay was done for the first time in 2012. It showed DSA against first donor (A 24-MFI 3711; $2^{\text {nd }}$ donor (DR15 MFI 1080); and against third donor (DQ5 MFI 14-19000). HLA-class II reactivity was directed against DQB1 except anti DQ2. Repeat SAB class I assay done in 2013 showed nearly threefold rise in MFI against donor allele A24. SAB class I was again tested in May 2014 also corroborated strong reactivity against HLA-A24 (Table 2).

It is not possible to evaluate the cumulative DSA against the first two donors as HLA-DQB1 typing of donors was not done and one can comment only on the basis of DRB1- DQB1 association. SAB class I and PRA specification (class I and II) indicate that there was a change in the donor's reactivity pattern which was no correlated with the class II SAB assay. The PRA class II phenotype bead assay showed DSA against second donor HLA-DRB1 alleles (Table 1) which explains the strongly positive LXM result.

\begin{tabular}{|c|c|c|c|c|}
\hline Test & Method & Date & Class I & Class II \\
\hline Recipient & R-SSO & $17 / 04 / 13$ & $A^{*} 02,{ }^{*} 03 ;{ }^{*} 18,{ }^{*} 40$ & DRB ${ }^{*} 07,{ }^{*} 11$ \\
\hline HLA Typing & SSP & $24 / 07 / 13$ & C*12 & DRB1*07,"11: DRB3, DRB4,DQB1*02,"03 \\
\hline $\begin{array}{l}\text { Recipient } \\
\text { HLA Typing }\end{array}$ & R-SSO & $07 / 03 / 14$ & $A^{*} 02,{ }^{*} 03 ;{ }^{*} 18,{ }^{*} 40$ & $\begin{array}{l}\text { DOA1*02:01, }{ }^{*} 05: ; \mathrm{DQB} 1{ }^{*} 02: 02,{ }^{*} 03 \\
\text { DPA1.01:03, }{ }^{*} 02: 01 ; \mathrm{DPB} 1{ }^{*} 04: 01,{ }^{*} 26: 01\end{array}$ \\
\hline $\begin{array}{l}\text { Donor } 3 \\
\text { HLA Typing }\end{array}$ & R-SSO & 07/03/14 & $\mathrm{A}^{*} 02,{ }^{*} 33 ; \mathrm{B}^{*} 44,{ }^{*} 50 \mathrm{C}^{*} 06,07$ & $\begin{array}{l}\text { DRB1 }{ }^{*} 01,1^{*} 07, \text { DRB4 } \\
\text { DQA } 1^{*} 01,{ }^{*} 02: 01 ; \mathrm{DQB} 1 * 02 ; 02^{*} 05 \\
\text { DPA } 1^{*} 01: 03,-; \mathrm{DPB} 1^{*} 02: 01,{ }^{*} 04: 02\end{array}$ \\
\hline Crossmatch & CDC-AHG & $27 / 02 / 14$ & B \& T cell negative & - \\
\hline Cross-match (MFI) & Luminex & $27 / 05 / 13$ & 151 & 577 \\
\hline- & Luminex & $27 / 02 / 14$ & 499.5 & 733 \\
\hline- & Luminex & $12 / 06 / 14$ & 210 & 346 (post Bortezomib addition). \\
\hline Post-transplant & Luminex & $17 / 07 / 14$ & 130 & 452 \\
\hline SAB- DSA (MFI) & Luminex & $27 / 02 / 14$ & Negative (<self) & Not done \\
\hline
\end{tabular}

Table 2: HLA typing results of recipient $3^{\text {rd }}$ donor and antibody workup.

Serial evaluation for DSA was done on basis of LXM with the third donor who was initially weakly positive even though the recipient had received plasmapheresis and potent immunosuppression. On addition of Bortezomib he became LXM negative and LXM is negative on posttransplant follow up. No features of rejection were seen on allograft biopsy which showed non-specific interstitial inflammation without evidence of tubular necrosis. Patient initially tested positive for viremia but later became negative although viriuria still persists.

\section{Immunosuppression}

Desensitization protocol included Bortezomib, Campath and Rituximab in addition to plasmapheresis. He developed BK Virus nephropathy in the allograft after four months post-transplant, which improved with lowering of immunosuppression. Maintenance drugs include Leflunamide (20 mg), Wysolone (5 mg) in single daily dosage and Pan graph $(1 \mathrm{mg})$ twice daily. At four years post-transplant, the recipient has excellent renal function and continues to be DSA negative.

\section{Discussion}

Unlike in United Kingdom, there are no laid down guidelines for pre-transplant evaluation of prospective renal recipients in India [4]. At the time of this patient's work up SAB test had been commenced in very limited centres in the country and they probably had limited experience, so was not used widely [5]. HLA-DQB1 typing was not performed for the first two donors in spite of the fact that HLA-Class II DSA was detected against all the donors on LXM and on phenotype assay. Some studies have suggested that LXM detects only HLA-IgG 
Citation: Mahendra MN, Bansal I, Lal V (2018) Successful Renal Re-transplant in a Patient with Alport Syndrome: A Case Report. J Kidney 4:

Page 3 of 3

directed against HLA-DRB1 alleles [6]. Our findings however suggest that it may detect anti DQB1 antibodies, if the reactivity is very high, as can be inferred by the reactivity against first and third donors. The recipient had no DSA against discrepant DRB1 antigen in the third donor but showed class II LXM positivity even after administration of strong immunosuppression in addition to plasmapheresis which became negative three days prior to transplant. HLA-Class II phenotype assay results showed MFI of 3129 against HLA-DQ5 which can explain positive LXM results.

In the only other available report of HLAi renal transplant from India the DSA MFI was less than 2500 for all three patients, who were first time recipients and responded to single dose of Rituximab with plasmapheresis and [7]. The first SAB class II MFI against mismatched DRB1 antigens of the second donor (DR10 and DR15) which was $<1500$ albeit with very strong reactivity against the likely mismatched DQ allele (DQ6 or rarely DQ5). The possibility of masked reactivity against mismatched DRB1 alleles is another possibility, which would have become apparent on adding EDTA or testing in dilution $[8,9]$.

It is probably the first documented case of HLA incompatible renal re-transplant in India across HLA-DQB1 barrier. The other interesting aspect of this case is that even after BK Virus allograft nephropathy which was shown to be associated with graft loss in up to $46.1 \%$ of recipients and more likely in retransplants and with cytomegalovirus positivity as in the present patient [10]. At the time of submitting this case report the recipient has excellent renal function.

\section{Conclusion}

The case report is of a patient with Alport syndrome who received second renal transplant across HLA-DQB1 incompatibility as defined by positive pre-transplant LXM, went on to develop BK Virus allograft nephropathy from which he recovered and has excellent graft function.

\section{Acknowledgement}

I would like to acknowledge Mr Rakesh Kumar, Ms Anchal Arora for helping me with getting the data.

\section{References}

1. Levy M, Feingold J (2000) Estimating prevalence in single-gene kidney diseases progressing to renal failure. Kidney Int 58: 925-943.

2. Mallett A, Tang W, Clayton PA, Stevenson S, McDonald SP, et al.(2014) End-stage kidney disease due to Alport syndrome: Outcomes in 296 consecutive Australia and New Zealand dialysis and transplant registry cases. Nephrol Dial Transplant 29: 2277-2286.

3. Mishra MN, Lal V (2016) Luminex crossmatch as a successful algorithm for post-transplant follow-up in developing nations-A study of 169 consecutive samples. J Kidney 2: 127.

4. Guidelines for the detection and characterization of clinically relevant antibodies in allotransplantation, 2014.

5. Mishra MN, Lal V (2018) A novel strategy for donor specific antibody detection for haploidentical hematopoietic stem cell transplantation. Austin Transplant Sci 3: 1009.

6. Guillaume N, Mazouz H, Pio V, Presne C, Westeel PF (2013) Correlation between Luminex donor-specific crossmatches and levels of donorspecific antibodies in pretransplantation screening. Tissue Antigens 82: $16-20$.

7. Aggarwal G, Tiwari AK, Dorwal P, Chauhan R, Arora D, et al. (2017) Successful renal transplantation across HLA barrier: Report from India. Indian J Nephrol 27: 210-214.

8. Visentin J, Vigata M, Daburon S, Contin-Bordes C, Fremeaux-Bacchi V, et al. (2014) Deciphering complement interference in anti-human leukocyte antigen antibody detection with flow beads assays. Transplantation 98: 625-631.

9. Schnaidt $M$, Weinstock C, Jurisic M, Schmid-Horch B, Ender A, et al. (2011) HLA antibody specification using single-antigen beads--a technical solution for the prozone effect. Transplantation 92: 510-515.

10. Hirsch HH, Brennan DC, Drachenberg CB, Ginevri F, Gordon J, et al. (2005) Polyoma virus associated nephropathy in renal transplantation: Interdisciplinary analyses and recommendations. Transplantation 79: 1277-1286. 